\title{
The Effects of Powdered Fertilized Eggs on Depression
}

\author{
Ester Solberg \\ Department for Drug Abuse and Psychiatry, Blefjell Hospital, Kongsberg, Norway.
}

\begin{abstract}
This 12-week, double-blind, placebo-controlled study investigated the effects of fertilized egg powder (Young Tissue Extract; YTE $^{\circledR}$ ) intake on outcome measures for depression. Fifty-five patients with depression were randomly assigned to receive YTE, YTE plus Melissa officinalis, or placebo for 12 weeks. At baseline, there were no significant differences in scores on the Hamilton Depression Rating Scale (HAM-D) or Beck Depression Inventory II (BDI-II) among the 3 groups. At 12 weeks, the HAM-D scores in groups treated with YTE or YTE with M. officinalis were both significantly lower than those in the placebo group. In addition, both treatment groups showed a significant improvement in depression as measured by the change in HAM-D scores from baseline to 12 weeks, whereas the placebo group showed no significant change. There were no significant differences between the 2 treatment groups. The study indicates that the fertilized egg powder has an antidepressive effect and may be an alternative or adjunct to antidepressive medication for some patients, but further research is necessary.
\end{abstract}

\section{KEY WORDS: • depression $\bullet$ egg $\bullet$ emotional symptoms $\bullet$ nutritional supplement $\bullet$ somatoform symptoms $\bullet$ Young Tissue Extract}

\section{INTRODUCTION}

D EPRESSION AFFECTS APPROXIMATELY 121 million people worldwide and is the fourth leading contributor to the global burden of disease. ${ }^{1}$ Estimates of the lifetime prevalence of depression vary, but approximately $17 \%$ of the population will experience a depressive episode. ${ }^{2}$

The most widely used pharmaceutical treatments for depression are selective serotonin reuptake inhibitors. However, not all depressed patients respond to these agents or to other pharmaceuticals. ${ }^{3}$ An estimated $29 \%-46 \%$ of patients with major depressive disorder do not respond or only partially respond to treatment with antidepressant medication. ${ }^{4}$ There has been growing interest in complementary and alternative medicine for a variety of medical conditions, including depression. ${ }^{3,5,6}$ Approximately $44 \%$ of psychiatric inpatients have used an herbal preparation to treat a variety of symptoms. ${ }^{7}$

Egg components have a variety of physiologic effects, including novel antimicrobial, immunomodulatory, anticancer, and antihypertensive activities; antioxidant properties; protease-inhibiting ability; and nutrient bioavailability. ${ }^{8}$ The isolate investigated in the current study, Young Tissue Extract (YTE ${ }^{\circledR}$; Med-Eq AS, Tonsberg, Norway), is extracted from fertilized, partially incubated hen eggs. Two pilot

Manuscript received 7 June 2010. Revision accepted 17 November 2010.

Address correspondence to: Ester Solberg, Olav Duunsvei 15, 3080 Holmestrand, E-mail: est-skr@online.no studies, 1 published ${ }^{9}$ and 1 unpublished* have reported that YTE improves libido in healthy adults and in adults taking antidepressant medication. A recently published study reported that 4 weeks of YTE use $(1,680 \mathrm{mg} / \mathrm{d})$ decreased the anxiety response to a laboratory stressor in participants experiencing chronic stress. ${ }^{10}$ A 3-week preliminary study of YTE for treatment of sexual dysfunction in participants taking an antidepressant medication revealed significant improvement in self-reported mood.* Another preliminary study ${ }^{\dagger}$ reported significant improvement in depression scores on the Montgomery and Asberg Depression Rating Scale in participants taking YTE, Melissa officinalis, or a combination of both. The current investigation aimed to complete a preliminary randomized, controlled trial of the effectiveness of YTE or a combination of YTE and Melissa officinalis on depression.

M. officinalis (lemon balm), derived from the dried leaves of the plant by the same name and containing volatile oils (citronellal, geranial, and neral) ${ }^{11}$ has been used in herbal medicine for many centuries. Common uses of this herb include treating insomnia. It may also have anxiolytic and calming properties. ${ }^{12-14}$ Therefore, the current study sought to investigate whether this herb could boost the effects of YTE on depression, given its effectiveness in a pilot study.

\footnotetext{
*Törnblom K: The effect of "libido" on decreased sexual desire associated with antidepressant medication. Unpublished article. Med-Eq AS, Tønsberg, Norway, 1997.

'Solberg E: Pilot study on Vivax. Unpublished article. Med-Eq AS, Tønsberg, Norway, 2010 .
} 


\section{MATERIALS AND METHODS}

\section{Protocol}

The study was a randomized, placebo-controlled, doubleblind study examining the effect of YTE or YTE in combination with $M$. officinalis for depression.

\section{Participants}

Of the 66 participants, 45 women and 10 men completed the study, and 11 participants dropped out. Participants were recruited through advertising in local newspapers in the county of Vestfold, Norway. All participants provided signed written informed consent before entering the study. The regional ethics committee was notified, and the study was conducted according to the principles of the Declaration of Helsinki, good clinical practice, and local regulations.

Main outcomes were change in score on the Hamilton Depression Rating Scale (HAM-D) and the Beck Depression Inventory II (BDI-II) over the 12-week period. ${ }^{15,16} \mathrm{~A}$ psychologist administered both the BDI-II and the HAMDD. Inclusion criteria were a minimum BDI-II score of 10. Exclusion criteria were risk for suicide; serious mental disorders; current use of antidepressive agents, benzodiazepines, or pain killers with codeine; and alcohol or drug addiction. Six participants used low-dose medication or other occasional herbal preparations, and they continued their medication through the study. Participants were randomly assigned to the treatment groups.

Four participants dropped out during the study: One participant dropped out to seek additional treatment for depression, 1 reported irregular heartbeat and wished to discontinue medication, 1 discontinued medication because of stomach pain, and 1 participant was unreachable for the last consultation. For the final consultation, 3 treatment group participants and 2 placebo group participants were interviewed via telephone for the BDI-II. HAM-D interviews were not conducted for these participants. Two participants violated study protocol and were excluded from the study.

\section{Investigational treatment}

An isolate (YTE) from fertilized hen eggs has been developed. Fertilized eggs are placed in an incubator for 9 days; the embryonic portion is mechanically separated from any remaining liquid. The isolate is then sterilized, deep-frozen for a minimum of 2 days, and freeze-dried at $56^{\circ} \mathrm{C}$ for 4 hours. One capsule contains $336 \mathrm{mg}$ of the isolate. One study group received 5 capsules per day, corresponding to $1,680 \mathrm{mg}$ of YTE; another group received 1,680 mg of YTE in addition to $600 \mathrm{mg}$ of M. officinalis (120 mg per capsule); and the placebo group received corresponding amounts of lactose. The 3 different capsules were stored in separate, unmarked, sealed containers, and the text instructed participants to ingest 3 capsules every morning and 2 every evening.

\section{Baseline}

The participants met for consultation before inclusion in the study and after 3, 6, and 12 weeks of YTE administra- tion. The BDI-II was administered at every consultation, whereas the HAM-D was administered only at baseline and after 12 weeks. Treatment adherence was monitored during follow-up consultations. Participants returned study pill bottles, and the remaining number of capsules was counted at each consultation.

\section{Statistical analysis}

All values are expressed as means \pm standard deviation unless otherwise stated. Comparisons of baseline characteristics among the 3 were performed by using analysis of variance (ANOVA) for continuous variables and a $\chi^{2}$ test for the categorical variable, sex. Changes in both HAM-D and BDI-II scores from baseline to 12 weeks were examined by using ANOVA along with the Tukey-Kramer method to test the significance of pairwise group comparisons. BDI-II scores at baseline and 3,6, and 12 weeks and HAM-D scores at baseline and 12 weeks were examined by using repeated-measures ANOVA and the Bonferroni method. When the assumption of normality seemed to be violated, a nonparametric procedure (Kruskal-Wallis test) was used to test the differences among the 3 groups. For hypothesis-generating purposes, both treatment groups were pooled (YTE only and the combination treatment) and compared with the placebo group by using the same statistical analyses performed with the separate treatment groups. $P$ values less than .05 were considered to indicate a statistically significant difference. All statistical analyses were performed by using SAS statistical software, version 8.2 (SAS Institute Inc., Cary, North Carolina, USA).

\section{RESULTS}

\section{Participants}

Table 1 lists baseline demographic and clinical measures. The 3 treatment groups were tested for the homogeneity of demographic variables and other baseline measures (e.g., sex, age, baseline BDI and HAM-D scores). Demographic variables and baseline HAM-D or BDI-II scores did not significantly differ between groups.

\section{Investigational treatments}

After 12 weeks of YTE, both treatment groups had significantly lower scores on the HAM-D compared with

Table 1. Demographic and Clinical Characteristics of Participants at Baseline

\begin{tabular}{lccc}
\hline Characteristic & $\begin{array}{c}Y T E \\
(\mathrm{n}=18)\end{array}$ & $\begin{array}{c}Y T E+\text { M. officinalis } \\
(\mathrm{n}=19)\end{array}$ & $\begin{array}{c}\text { Placebo } \\
(\mathrm{n}=18)\end{array}$ \\
\hline Men/women (n/n) & $4 / 14$ & $3 / 16$ & $3 / 15$ \\
Age (y) & $46.2 \pm 8.1$ & $45.3 \pm 10.9$ & $44.8 \pm 14.2$ \\
BDI-II score & $22.6 \pm 7.5$ & $22.1 \pm 9.0$ & $20.6 \pm 1.2$ \\
HAM-D score & $19.5 \pm 5.7$ & $18.6 \pm 5.9$ & $18.4 \pm 3.7$ \\
\hline
\end{tabular}

Data are reported as mean \pm standard deviation.

BDI-II, Beck Depression Inventory II; HAM-D, Hamilton Depression Rating Scale; YTE, Young Tissue Extract. 
Table 2. Depression Measures During Treatment with Young Tissue Extract

\begin{tabular}{lccr}
\hline Variable & YTE & $Y T E+$ M. officinalis & Placebo \\
\hline BDI II & & & \\
Baseline & $22.6 \pm 7.5$ & $22.1 \pm 9.0$ & $20.6 \pm 5.2$ \\
Week 3 & $14.6 \pm 7.9$ & $15.2 \pm 10.4$ & $15.3 \pm 7.4$ \\
week 6 & $13.5 \pm 7.5$ & $13.0 \pm 9.5$ & $14.2 \pm 6.0$ \\
Week 12 & $11.9 \pm 7.7$ & $11.7 \pm 7.7$ & $16.1 \pm 8.4$ \\
Change from & $10.5 \pm 9.8$ & $11.0 \pm 9.3^{*}$ & $4.6 \pm 7.4$ \\
$\quad$ baseline & & & \\
HAM-D & & & \\
Baseline & $19.5 \pm 5.7$ & $18.6 \pm 5.9$ & $18.4 \pm 3.7$ \\
Week 12 & $9.4 \pm 4.5^{* *}$ & $10.3 \pm 5.0^{* *}$ & $16.5 \pm 6.5$ \\
Change from & $10.1 \pm 6.4^{* *}$ & $8.4 \pm 7.4^{* *}$ & $1.9 \pm 4.9$ \\
$\quad$ baseline & & & \\
\hline
\end{tabular}

Data are reported as mean \pm standard deviation.

$* P<.10$ compared with placebo

$* * P<.01$ compared with placebo.

placebo $(P<.01)$ (Table 2, Fig. 1). However, there were no significant differences between the group receiving only YTE and the combination treatment group. Both treatment groups showed significant improvement in mean scores (51.7\% for the YTE group and $44.8 \%$ for the YTE $+M$. officinalis group) when change in depression scores (baseline compared with 12 weeks) were evaluated $(P<.01)$. Similarly, the 2 treatment groups were not significantly different from each other.

BDI-II scores for the treatment groups were not significantly different when compared with placebo at any time point. Average BDI-II scores for the YTE group at week 3 $(P=1.0)$, week $6(P=1.0)$, and week $12(P=.49)$ did not significantly differ from those in the placebo group, nor did the average BDI-II scores for the YTE $+M$. officinalis group at week $3(P=1.0)$, week $6(P=1.0)$, and week 12 $(P=.31)$ (Fig. 2). However, compared with the placebo group, there was a nonsignificant trend for improvement from baseline to 12 weeks in the YTE $+M$. officinalis group $(P<.10)$. The 2 treatment groups did not significantly differ.

\section{Pooled analysis}

Because of the small sample size and the exploratory nature of the current experiment, the data from the 2 treatment groups were pooled into 1 data set and compared with placebo (Table 3). When the combined treatment group was compared with the placebo group, a significant difference in HAM-D scores was seen at 12 weeks $(P<.001)$. Treatment resulted in a $48.2 \%$ reduction in mean HAM-D scores from baseline to 12 weeks, resulting in significant improvment in HAM-D scores over the course of the study $(P<.001)$. There was a nonsignificant trend for BDI-II scores to be lower after 12 weeks of treatment compared with the placebo $(47.2 \%$ vs. $21.9 \%$, respectively), and the difference between the treatment and placebo groups at 12 weeks approached significance $(P<.10)$.

\section{DISCUSSION}

Twelve weeks of daily treatment with YTE or YTE with M. officinalis improved depression scores on the HAM-D compared with placebo. There was also a significant improvement in depression as measured in change from baseline on the HAM-D. No significant differences was observed between the 2 treatment groups.

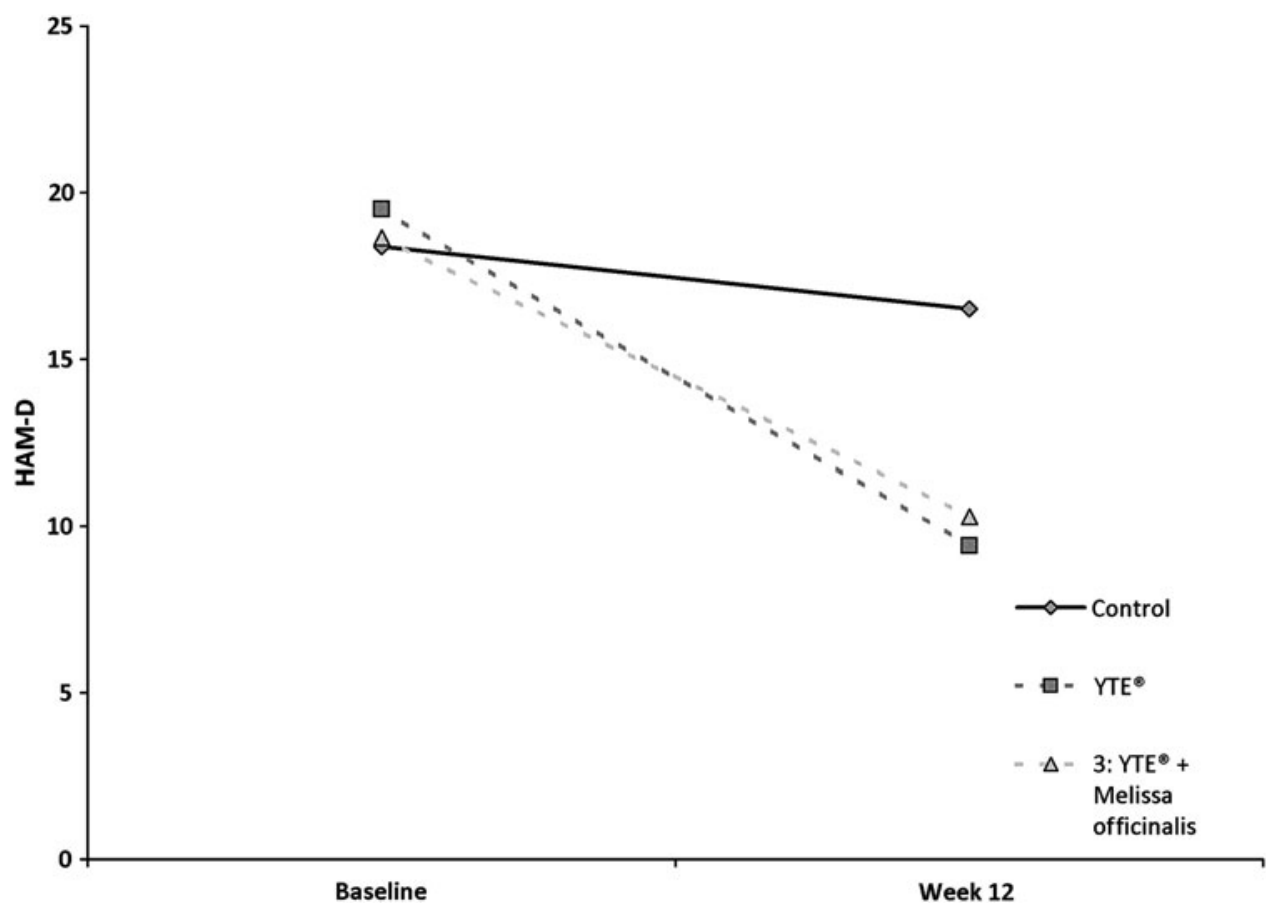

FIG. 1. A repeated-measures analysis of variance revealed a significant effect of Young Tissue Extract (YTE) or YTE with $M$. officinalis compared with placebo $(P=.017)$, as measured by scores on the Hamilton Depression Rating Scale. Study week also had an effect $(P=.002)$, with a decrease in depression score at week 12 compared with baseline. HAM-D, Hamilton Depression Rating Scale. 


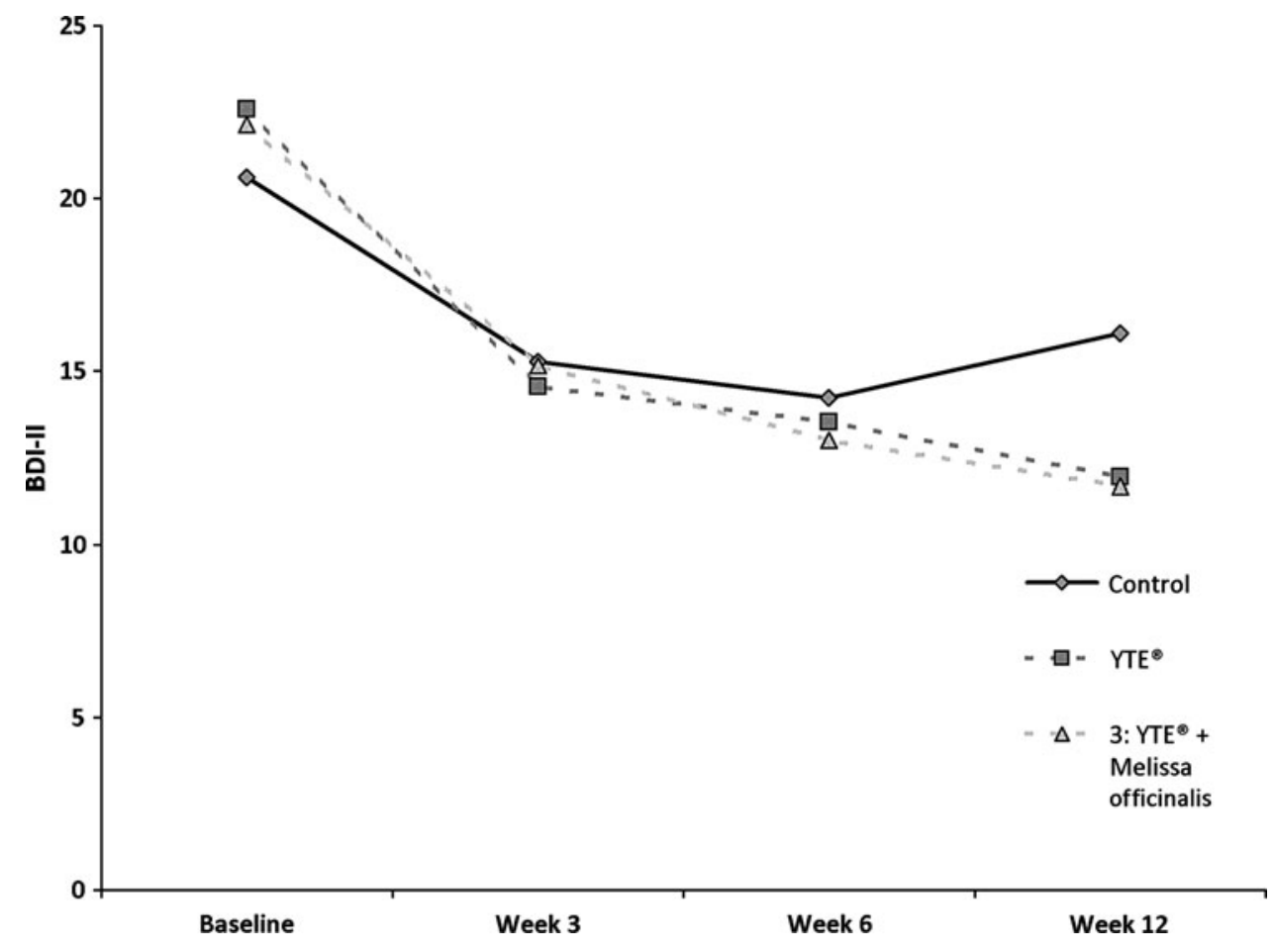

FIG. 2. Repeated-measures analysis of variance showed no significant effects of Young Tissue Extract (YTE) or YTE with Melissa officinalis, as measured by scores on the Beck Depression Inventory II $(P>.05)$, and showed no significant effect of study week $(P>.05)$. BDI-II, Beck Depression Inventory II.
There is growing interest in complementary and alternative medicine for the treatment of depression. ${ }^{17}$ Other herbal preparations that show some efficacy in treating depression include S-adenosyl-methionine (SAMe) and St. John's wort. ${ }^{5}$ The relationship between SAMe and depression has been studied for several decades. ${ }^{3}$ SAMe is suggested to be an effective depression treatment that is comparable to tricyclic antidepressants, with quicker action and fewer adverse effects. ${ }^{18,19}$ These findings suggest that there may be a variety of alternative methods for the treat-

Table 3. Pooled Analysis of Young Tissue Extract and Young Tissue Extract with MELISSa officinalis Compared with Placebo

\begin{tabular}{lcr}
\hline & YTE only and & \\
Variable & YTE + M. officinalis & Placebo \\
\hline BDI-II score & & \\
Baseline & $22.4 \pm 8.2$ & $20.6 \pm 5.2$ \\
Week 3 & $14.9 \pm 9.1$ & $15.3 \pm 7.4$ \\
Week 6 & $13.3 \pm 8.5$ & $14.2 \pm 6.1$ \\
Week 12 & $11.8 \pm 7.6^{*}$ & $16.1 \pm 8.4$ \\
Change from baseline & $10.8 \pm 9.4^{* *}$ & $4.6 \pm 7.4$ \\
HAM-D score & & \\
Baseline & $19.5 \pm 5.7$ & $18.4 \pm 3.7$ \\
Week 12 & $9.9 \pm 4.7^{* * *}$ & $16.5 \pm 6.5$ \\
Change from baseline & $9.2 \pm 6.8^{* * *}$ & $1.9 \pm 4.9$ \\
\hline
\end{tabular}

Data are reported as mean \pm standard deviation.

$* P<.10$ compared with placebo.

$* * P<.05$ compared with placebo.

$* * * P<.01$ compared with placebo. ment for depression. The chemical composition of YTE is unknown, and the mechanisms through which YTE may have antidepressant action are unidentified at this time.

The data collected on the BDI-II were grouped into questions that relate to 4 categories-vitality, low mood, vegetative symptoms, and cognitive depressive symptoms - to explore the relationship between YTE and depression. The HAM-D was divided into 4 categories: vitality, unrest and somatization, mood disorders, and vegetative symptoms (Table 4). This factorial analysis was done only to explore potential routes for further investigations, and it does not represent a validated method. These results suggest that the relationship between YTE and factors involving vitality, mood enhancement, and somatization warrant further research. There were no significant differences in measurements for vegetative or cognitive symptoms on the BDI-II or measurements for vegetative symptoms on the HAM-D.

YTE may have a beneficial effect on subjective stress and the endocrine stress response. ${ }^{10}$ YTE elevates 17-ketosteroid levels in the adrenal glands, which decreases stressrelated hormones such as cortisol. Dysregulation of the stress response may play a role in major depression. ${ }^{20} \mathrm{De}$ termination of any relationship between YTE and the stress response requires further research.

M. officinalis has calming and anxiolytic properties. The combination treatment, YTE with M. officinalis, had no additional effects compared with YTE alone. Both treatments significantly improved scores on the HAM-D after 12 weeks of treatment. However, a nonsignificant trend indicated that change from baseline in scores on the 
Table 4. Categorical Analysis of Depression Symptoms

\begin{tabular}{lcr}
\hline & YTE only and & \\
Depression symptoms & $Y T E+$ M. officinalis & Placebo \\
\hline BDI-II categories & & \\
$\quad$ Vitality & $3.7 \pm 3.0^{*}$ & $1.1 \pm 2.4$ \\
Mood disorders & $1.4 \pm 1.8^{* *}$ & $0.1 \pm 1.6$ \\
Vegetative & $1.5 \pm 2.3$ & $1.0 \pm 1.7$ \\
$\quad$ Cognitive & $4.5 \pm 4.5$ & $2.1 \pm 3.7$ \\
HAM-D categories & & \\
Vitality & $1.9 \pm 1.9^{* *}$ & $0.5 \pm 1.4$ \\
Somatization & $2.8 \pm 2.2^{*}$ & $0.5 \pm 2.1$ \\
Mood disorders & $2.8 \pm 2.8^{*}$ & $-0.1 \pm 1.9$ \\
Vegetative & $1.4 \pm 2.1$ & $0.8 \pm 1.8$ \\
\hline
\end{tabular}

Data are reported as mean \pm standard deviation. The table represents 4 categories of BDI-II and 4 categories of HAM-D. The BDI-II categories are: vitality (loss of interest; feeling of uselessness; fatigue; lack of value, energy, concentration, and sexual lust); mood disorders (sadness, lack of joy, suicidal thoughts, and weeping); vegetative symptoms (restlessness, sleep disturbances, and appetite); and cognitive symptoms (pessimism, sense of defeat, guilt punishment, dislike of self, self-criticism, undecidedness, and irritability). The HAM-D categories are: vitality (interest and ability to act, energy, somatization and pain, sexual dysfunction); somatization (agitation, mental anxiety, hypochondria); mood disorders (depressive mood and weeping, feelings of guilt, suicidal thoughts); and vegetative symptoms (insomnia and sleep disorders, appetite and weight loss).

$* P<.01$ compared with placebo.

$* * P<.05$ compared with placebo.

BDI-II had a tendency to improve in the combination treatment group compared with placebo. Given this tendency, a well-designed study with a larger sample size should investigate the hypothesis that a combination of YTE and M. officinalis is effective in treating depression. An additional trial administering $M$. officinalis alone should be done to rule out any benefits of the herb on depression.

As mentioned previously, preliminary studies suggest that YTE may influence mood. ${ }^{10, * \dagger}$ The results presented here extend those findings to suggest that YTE may influence depression measures. The current pilot study has several limitations. There was a small sample size $(n=55)$, and participants were not excluded if they used additional medications. The only depression diagnoses made at baseline were scores on the BDI-II, and adherence with the treatment protocol was not verified. These results should be verified by replication with a larger group of participants for a longer period. Given that another study administering fertilized egg powder has reported possible effects on the stress response, ${ }^{10}$ additional research should measure biological markers for the stress response. Similar to previous research on a variety of herbal preparations for the treatment of depression, it will also be necessary to compare YTE against a clinically verified antidepressant medication.

*Törnblom, unpublished.

${ }^{\dagger}$ Solberg, unpublished.

\section{ACKNOWLEDGMENTS}

Blefjell Hospital and Med-Eq AS, Tønsberg, Norway, provided financial support for this study.

\section{AUTHOR DISCLOSURE STATEMENT}

No competing financial interests exist.

\section{REFERENCES}

1. World Health Organization: Depression. www.who.int/topics/depression/en (Accessed May 5, 2010)

2. Pincus HA, Pettit AR: The societal costs of chronic major depression. J Clin Psychiatry 2001;62 Suppl 6:5-9.

3. Papakostas GI, Alpert JE, Fava M: S-adenosyl-methionine in depression: a comprehensive review of the literature. Curr Psychiatry Rep 2003;5:460-466.

4. Fava M, Davidson KG: Definition and epidemiology of treatment-resistant depression. Psychiatr Clin North Am 1996;19: 179-200.

5. Lavretsky H: Complementary and alternative medicine use for treatment and prevention of late-life mood and cognitive disorders. Aging Health 2009;5:61-78.

6. Deligiannidis KM, Freeman MP: Complementary and alternative medicine for the treatment of depressive disorders in women. Psychiatr Clin North Am 2010; 33:441-463.

7. Elkins G, Rajab MH, Marcus J: Complementary and alternative medicine use by psychiatric inpatients. Psychol Rep 2005;96: 163-166.

8. Kovacs-Nolan J, Phillips M, Mine Y: Advances in the value of eggs and egg components for human health. J Agric Food Chem 2005;53:8421-8431.

9. Eskeland B, Thom E, Svendsen KO: Sexual desire in men: effects of oral ingestion of a product derived from fertilized eggs. $J$ Int Med Res 1997;25:62-70.

10. Schult J, Hero T, Hellhammer J: Effects of powdered fertilized eggs on the stress response. Clin Nutr 2010;29:255-260.

11. Wheatley, D: Medicinal plants for insomnia: a review of their pharmacology, efficacy and tolerability. $J$ Psychopharmacol 2005;19:414-421.

12. Kennedy DO, Scholey AB, Tildesley NT, Perry EK, Wesnes KA: Modulation of mood and cognitive performance following acute administration of Melissa officinalis (lemon balm). Pharmacol Biochem Behav 2002;72:953-964.

13. Kennedy DO, Wake G, Savelev S, et al.: Modulation of mood and cognitive performance following acute administration of single doses of Melissa officinalis (lemon balm) with human CNS nicotinic and muscarinic receptor-binding properties. $\mathrm{Neu}$ ropsychopharmacology 2003;28:1871-1881.

14. Kennedy DO, Little W, Scholey AB: Attenuation of laboratoryinduced stress in humans after acute administration of Melissa officinalis (lemon balm). Psychosom Med 2004;66:607-613.

15. Hamilton M: A rating scale for depression. J Neurol Neurosurg Psychiatry 1960;23:56-62.

16. Beck A, Steer R, Brown G: The Beck Depression Inventory. San Antonio, Texas, USA, Psychological Corp., 1993.

17. Meeks TW, Wetherell JL, Irwin MR, Redwine LS, Jeste DV: Complementary and alternative treatments for late-life depression, anxiety, and sleep disturbance: a review of randomized controlled trials. J Clin Psychiatry 2007;68:1461-1471. 
18. Delle Chiaie R, Pancheri P, Scapicchio P: Efficacy and tolerability of oral and intramuscular S-adenosyl-L-methionine 1,4butanedisulfonate (SAMe) in the treatment of major depression: comparison with imipramine in 2 multicenter studies. Am J Clin Nutr 2002;76:1172S-1176S.
19. Mischoulon D, Fava M: Role of S-adenosyl-L-methionine in the treatment of depression: a review of the evidence. Am J Clin Nutr 2002;76:1158S-1161S.

20. Claes S: Glucocorticoid receptor polymorphisms in major depression. Ann N Y Acad Sci 2009;1179:216-228. 